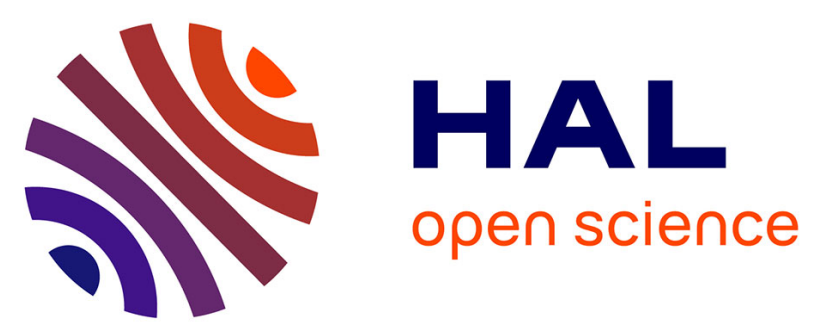

\title{
A 3D insect-inspired visual autopilot for corridor-following
}

Geoffrey Portelli, Julien Serres, Franck Ruffier, Nicolas Franceschini

\section{To cite this version:}

Geoffrey Portelli, Julien Serres, Franck Ruffier, Nicolas Franceschini. A 3D insect-inspired visual autopilot for corridor-following. EMBS International Conference on Biomedical Robotics and Biomechatronics (BioRob 2008), Oct 2008, Scottsdale, United States. pp.19-26, 10.1109/BIOROB.2008.4762816 . hal-02294590

\section{HAL Id: hal-02294590 https://hal-amu.archives-ouvertes.fr/hal-02294590}

Submitted on 23 Sep 2019

HAL is a multi-disciplinary open access archive for the deposit and dissemination of scientific research documents, whether they are published or not. The documents may come from teaching and research institutions in France or abroad, or from public or private research centers.
L'archive ouverte pluridisciplinaire HAL, est destinée au dépôt et à la diffusion de documents scientifiques de niveau recherche, publiés ou non, émanant des établissements d'enseignement et de recherche français ou étrangers, des laboratoires publics ou privés. 


\title{
An Insect-Inspired Visual Autopilot for Corridor-Following
}

\author{
Geoffrey Portelli, Julien Serres, Franck Ruffier, and Nicolas Franceschini
}

\begin{abstract}
Motivated by the results of behavioral studies performed on bees over the last two decades, we have attempted to decipher the logics behind the bee's autopilot, with specific reference to their use of optic flow (OF). Using computer-simulation experiments, we developed a vision-based autopilot that enables a 'simulated bee' to travel along a tunnel by controlling both its speed and its clearance from the walls, the ground, and the ceiling. The flying agent is fully actuated and can translate along three directions: surge, sway, and heave. The visuo-motor control system, called ALIS (AutopiLot using an Insect based vision System), is a dual OF regulator consisting of intertwined feedback loops, each of which has its own OF set-point. The experiments show that the simulated bee navigates safely along a straight or tapered tunnel and reacts sensibly to major OF perturbations caused, e.g., by the lack of texture on one wall or by the presence of a tapered tunnel. The agent is equipped with a minimalistic visual system (comprised of only eight pixels) that suffices to control the clearance from the four walls and the forward speed jointly, without the need to measure any speeds and distances. The OF sensors and the simple visuo-motor control system developed here are suitable for use on MAVs with avionic payloads as small as a few grams. Besides, the ALIS autopilot accounts remarkably for the quantitative results of ethological experiments performed on honeybees flying freely in straight or tapered corridors.
\end{abstract}

\section{ACRONYMS}

ALIS AutopiLot using an Insect-based vision System

AUV Autonomous Underwater Vehicles

DOF Degree Of Freedom

EMD Elementary Motion Detector

LORA III Lateral Optic flow Regulation Autopilot III [1]

MAV Micro-Air Vehicle

OCTAVE Optical flow based Control Syst. for Aerial Vehicles [2]

OF Optic Flow

ROV Remotly Operated Vehicle

\section{INTRODUCTION}

$\mathrm{W}$ inged insects are able to navigate in unfamiliar environments by relying heavily on the optic flow (OF) [3] that is generated by their own motion [4]. Insects rely on OF to avoid lateral obstacles [5-6], to control

Manuscript received on April 22, 2008.

This work was supported partly by CNRS (Life Science; Information and Engineering Science and Technology), by the Univ. of the Mediterranean, by an EU contract (IST/FET -1999- 29043), by the French Agency for Research (ANR, RETINAE project) and by the French Defense Agency (DGA, 0534 022).

G. Portelli, F. Ruffier, J. Serres and N. Franceschini are with the Biorobotics Dept. at the Institute of Movement Sciences, CNRS / Uni. of the Mediterranean, CP938, 163 ave. Luminy, 13288 Marseille Cedex 09, France (phone: +33 491828366 ; fax +33 4918283 75; e-mail:

geoffrey.portelli@univmed.fr, franck.ruffier@univmed.fr,

julien.serres@univmed.fr, nicolas.franceschini@univmed.fr). their speed [7-9] and height [10-11], to cruise and land [8,11-12].

Honeybees trained to fly in a narrow tunnel were observed to fly close to the midline [5]. To explain this centering response, these authors hypothesized that the animal may balance the two lateral OFs. Several roboticists have designed visually guided vehicles based on this 'OF balance' hypothesis (e.g. [13-14]). Some added a speed control system relying on the bilateral OF [15-17].

Recently, we developed the LORA III autopilot based on a pair of lateral OF regulators for a fully actuated hovercraft, in which surge and sway dynamics are uncoupled [1]. The LORA III autopilot was shown to account remarkably well for behaviors (including centering and wall-following behaviors) observed in bees flying along stationary or nonstationary corridors [5-6] as well as tapered corridors [8].

The ALIS autopilot described here extends the LORA III autopilot principle to the vertical plane. Here, the issue is to establish a functional diagram of a joint speed control and obstacle avoidance system that would take into account not only lateral obstacles but also ventral obstacles [10-11], as well as dorsal obstacles. The ALIS autopilot we arrived at allowed us to test a simulated honeybee, in which all the translational DOF (surge, sway, and heave) are known to be uncoupled [18]. This simulation therefore extends the existing 2D model [1] to $3 \mathrm{D}$ and entails the following novel features:

-- regulating (i.e. maintaining constant) the vertical OF (i.e., the OF perceived ventrally and dorsally) via the surge or heave dynamics,

-- using walls, ground, and ceiling that are all textured with natural scenes,

-- using a 2D model of photoreceptor sensitivity.

The ALIS autopilot regulates the OF by both positioning and forward control systems, according to the following principles:

1) the first OF regulator adjusts the bee's forward speed so as to keep the maximum of the sum of the two opposite OFs equal to a forward OF set-point. The outcome is that the bee's forward speed will become proportional to the narrower dimension (either width or height) of the flight tunnel. The value of the forward speed attained will meet the forward OF set-point.

2) the second OF regulator adjusts the bee's lateral or vertical position so as to keep the maximal OF equal to the positioning OF set-point. The outcome is that the clearance from the nearest substrate (walls, ground, or ceiling) will become proportional to the bee's forward speed as defined in 1). The clearance from the nearest substrate will meet the positioning OF set-point. 
The ALIS autopilot enables the agent to perform obstacle avoidance using maneuvers that involve translational DOF exclusively, unlike obstacle avoidance based on body saccades that involve rotational DOFs [19-22]. The ALIS autopilot operates by measuring neither speed nor distance, and therefore differs fundamentally from "insect-like" navigation involving speed regulation and distance regulation [23].

In section II, we describe the dynamical model of the simulated bee along its three translational DOFs. In section III, the simulation set-up used to test the ALIS autopilot onboard the simulated bee is described. Section IV describes the ALIS autopilot in detail. Section V shows the results of computer-simulated experiments carried out on the simulated bee, which is able to perform various tasks such as takeoff, straight or tapered tunnel-following, and to react sensibly to the local absence of lateral or dorsal OF.

\section{II.DYNAMICAL MODEL OF BEE FLIGHT}

Here, we focus on the visuomotor feedback loops that may explain how a flying insect can control its speed and avoid obstacles. We propose a linearized model of the bee's dynamics along the 3 translational DOFs (surge, sway, and heave dynamics). Linearization is justified by the limited range of speed $(0-2 \mathrm{~m} / \mathrm{s})$ that is considered here. We maintain the 3 rotational DOFs at a zero value because bees are known to be equipped with a heading lock system [24] that make them fly straight to the nectar source [25]. Moreover, the simulated insect is not subjected to any wind: the groundspeed is taken to be equal to the airspeed. We detail below the bee's dynamics along its 3 translational DOFs.

\section{A. Surge dynamics}

Experiments on fruitflies [26], and honeybees [27-28], have shown that flying insects gain forward speed by pitching forward their mean flight-force vector $F$ at a small angle $\theta_{\text {pitch }}\left(\sim 20^{\circ}\right)$ with respect to the vertical (Fig. 1a,b). Minute change in the insect wing stroke plane angle $\theta_{\text {pitch }}$ provides forward thrust $T$, while hardly affecting the vertical lift $L$ [18].

\section{B. Sway dynamics}

In flying insects like bees, sideslip motion results from roll changes [18]. The wing stroke plane roll angle $\theta_{\text {roll }}$ therefore drives the side thrust $S$ (Fig. 1a,c).

\section{Heave dynamics}

The mean flight-force $F$ resulting from the wing stroke amplitude $\Phi$ [29-30] can be resolved in forward Thrust $T$ (1a), Side thrust $S(1 \mathrm{~b})$, and vertical Lift $L$ (1c). At small angles $\left(\theta_{\text {pitch }}\right.$ and $\left.\theta_{\text {roll }}\right), L$ is roughly equal to $F$. The wing stroke amplitude $\Phi$ therefore mainly drives the vertical lift $L$.

$T=F(\Phi) \cdot \sin \left(\theta_{\text {pitch }}\right) \cdot \cos \left(\theta_{\text {roll }}\right)$

$S=F(\Phi) \cdot \cos \left(\theta_{\text {pitch }}\right) \cdot \sin \left(\theta_{\text {roll }}\right)$

$L=F(\Phi) \cdot \cos \left(\theta_{\text {pitch }}\right) \cdot \cos \left(\theta_{\text {roll }}\right)$

In order to determine the gain $K_{\text {wing }}$ between the lift and the wing stroke plane amplitude, we can refer to experiments on

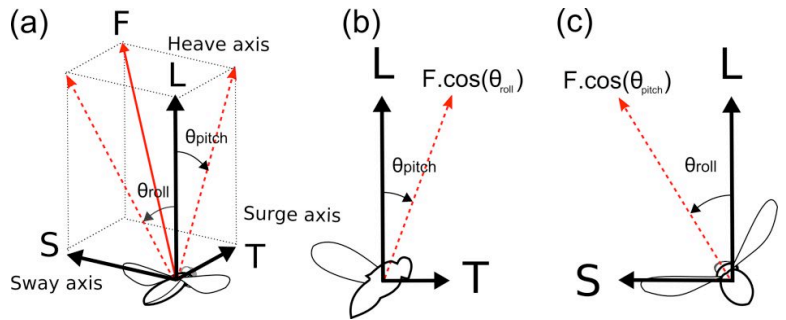

Fig. 1. (a) Resolution of the mean flight-force vector $F$ along the surge $\mathrm{X}$-axis giving the forward thrust $T$, along the sway $\mathrm{Y}$-axis giving the side thrust $S$, and along the heave $\mathrm{Z}$-axis giving the vertical lift $L$. (b) Pitching the mean flight-force vector $F$ by an angle $\theta_{\text {pitch }}$ generates a forward thrust $T$. (c) Rolling the mean flight-force vector $F$ by an angle $\theta_{\text {roll }}$ generates a side thrust $S$.

hovering bees that were carried out in media of different densities. Hovering bees have been filmed in normal air $\left(\rho_{\text {Air }}=1,21 \mathrm{~kg} / \mathrm{m}^{3}\right)$ and in heliox $\left(\rho_{\text {Heliox }}=0,41 \mathrm{~kg} / \mathrm{m}^{3}\right)$ [30]. In the low density heliox, bees were observed to increase their wing stroke amplitude $\Phi$ from $90^{\circ}$ to $130^{\circ}$, while keeping the wingbeat frequency constant. In these two hovering situations, the lift $L$ is equal to the weight: $L_{\text {Heliox }}\left(\Phi=130^{\circ}\right)$ $=L_{\text {Air }}\left(\Phi=90^{\circ}\right)=m \cdot g \approx 1 \mathrm{mN}$.

Production of bee's lift depends on both the density $\rho$ and the wing stroke amplitude $\Phi$ [31]. In a steady state analysis, at a given stroke amplitude $\Phi=130^{\circ}[18,32]$, the lift is proportional to the density:

$L_{\text {Air }}\left(\Phi=130^{\circ}\right) / \rho_{\text {Air }}=L_{\text {Heliox }}\left(\Phi=130^{\circ}\right) / \rho_{\text {Heliox }}$. We therefore compute $L_{A i r}\left(\Phi=130^{\circ}\right) \approx 3 \mathrm{mN}$, and derive the mean sensitivity $K_{\text {wing }}=\left(\partial L_{A i r} / \partial \Phi\right)=50 \mu \mathrm{N} /{ }^{\circ}$ when bees hover $\left(\Phi_{\text {Hover }}=90^{\circ}\right)$.

At small pitch $\left|\theta_{\text {pitch }}\right| \leq 20^{\circ}$ and roll $\left|\theta_{\text {roll }}\right| \leq 20^{\circ}$ angles, each component of the mean flight-force vector $F$ can be linearized along the surge (2a), sway (2b), and heave axes (2c) as a function of pitch angle $\theta_{\text {pitch }}$, roll angle $\theta_{\text {roll }}$, and stroke amplitude $\Phi=\Phi_{\text {Hover }}+\Delta \Phi$, respectively:

$T=m \cdot g \cdot \theta_{\text {pitch }}$

$S=m \cdot g \cdot \theta_{\text {roll }}$

$L=K_{\text {wing }} \cdot\left(\Phi_{\text {Hover }}+\Delta \Phi\right)$, with $K_{\text {wing }} \cdot \Phi_{\text {Hover }}=m \cdot g$

The following linearized system of equations referred to the bee's center of gravity is:

$m \cdot d V_{x} / d t+\zeta_{x} \cdot V_{x}=T=m \cdot g \cdot \theta_{\text {pitch }}$

$m \cdot d V_{y} / d t+\zeta_{y} \cdot V_{y}=S=m \cdot g \cdot \theta_{\text {roll }}$

$m \cdot d V_{z} / d t+\zeta_{z} \cdot V_{z}=L-m \cdot g=K_{\text {wing }} \cdot \Delta \Phi$

where $g$ is the gravity constant, $m=100 \mathrm{mg}$ (the bee's mass), and $\zeta_{\mathrm{x}}, \zeta_{\mathrm{y}}$, and $\zeta_{\mathrm{z}}$ the translational viscous friction coefficients along the $\mathrm{X}, \mathrm{Y}$, and Z-axis, respectively. This system of equations (3) can be also written as:

$\tau_{\text {Surge }} \cdot d V_{x} / d t+V_{x}=m \cdot g / \zeta_{x} \cdot \theta_{\text {pitch }}$

$\tau_{\text {Sway }} \cdot d V_{y} / d t+V_{y}=m \cdot g / \zeta_{y} \cdot \theta_{\text {roll }}$

$\tau_{\text {Heave }} \cdot d V_{z} / d t+V_{z}=K_{\text {wing }} / \zeta_{z} \cdot \Delta \Phi$

The bee's surge time constant $\tau_{\text {Surge }}=m / \zeta_{x}=0.22 \mathrm{~s}$ can be estimated from bees' landings data [12] and bee's OF based autopilot [11]. We assume here that the sway time constant and the heave time constant are the same as the surge time constant: $\tau_{\text {Surge }}=\tau_{\text {Sway }}=\tau_{\text {Heave }}=0.22 \mathrm{~s}$. The three 
translational viscous friction coefficients can therefore be computed: $\zeta_{\mathrm{x}}=\zeta_{\mathrm{y}}=\zeta_{\mathrm{z}}=0.455 \mathrm{mN} /(\mathrm{m} / \mathrm{s})$.

The sensitivity $K_{\text {Surge }}$ of forward speed $V_{x}$ versus pitch angle $\theta_{\text {pitch }}$ can be determined from figure 1(b) in [28] and estimated as $\left|\partial V_{x} / \partial \theta_{\text {pitch }}\right|=K_{\text {Surge }}=0.10(\mathrm{~m} / \mathrm{s}) /{ }^{\circ} . K_{\text {Sway }}$ is assumed to have a similar value.

$\tau_{\text {Surge }} \cdot d V_{x} / d t+V_{x}=K_{\text {Surge }} \cdot \theta_{\text {pitch }}$

$\tau_{\text {Sway }} \cdot d V_{y} / d t+V_{y}=K_{\text {Sway }} \cdot \theta_{\text {roll }}$

$\tau_{\text {Heave }} \cdot d V_{z} / d t+V_{z}=K_{\text {wing }} \cdot K_{\text {Heave }} \cdot \Delta \Phi$,

with $K_{\text {Heave }}=1 / \zeta_{z}=2200(\mathrm{~m} / \mathrm{s}) / \mathrm{N}$

The transfer functions for surge dynamics $G_{V x}(s)$ (6a), sway dynamics $G_{V y}(s)(6 \mathrm{~b})$, and heave dynamics $G_{V z}(s)(6 \mathrm{c})$ can therefore be written as follows:

$$
\begin{aligned}
& G_{V x}(s)=\frac{V x(s)}{\theta_{\text {pitch }}(s)}=\frac{K_{\text {Surge }}}{1+\tau_{\text {Surge }} \cdot s}=\frac{0.10}{1+0.22 \cdot s} \\
& G_{V y}(s)=\frac{V y(s)}{\theta_{\text {roll }}(s)}=\frac{K_{\text {Sway }}}{1+\tau_{\text {Sway }} \cdot s}=\frac{0.10}{1+0.22 \cdot s} \\
& G_{V z}(s)=\frac{V z(s)}{\Delta \Phi(s)}=\frac{K_{\text {Wing }} \cdot K_{\text {Heave }}}{1+\tau_{\text {Heave }} \cdot s}=\frac{0.11}{1+0.22 \cdot s}
\end{aligned}
$$

Here, the pitch angle is limited to $\left|\theta_{\text {pitch }}\right| \leq 20^{\circ}$ so as to limit the forward speed range at $V_{x \max }=2 \mathrm{~m} / \mathrm{s}$ and the roll angle is limited to $\left|\theta_{\text {roll }}\right| \leq 5^{\circ}$ so as to limit the lateral speed range at $V_{\text {ymax }}=0.5 \mathrm{~m} / \mathrm{s}$. Bees apparently reach a maximum stroke amplitude $\Phi_{\operatorname{Max}}=140^{\circ}$ and a minimum stroke amplitude $\Phi_{\text {Min }}=70^{\circ}$ [29,33]. They hover with a stroke amplitude $\Phi_{\text {Hover }}=90^{\circ}$. Then, the maximum ascent speed $V_{z U p M a x}$ and the maximum descent speed $V_{z \text { DownMax }}$ along the heave-axis are:

$V_{z \text { ZPMax }}=K_{\text {wing }} \cdot K_{\text {Heave }} \cdot\left(\Phi_{\text {Max }}-\Phi_{\text {Hover }}\right)=5.5 \mathrm{~m} / \mathrm{s}$

$V_{\text {zDownMax }}=K_{\text {wing }} \cdot K_{\text {Heave }} \cdot\left(\Phi_{\text {Hover }}-\Phi_{\text {Min }}\right)=2.2 \mathrm{~m} / \mathrm{s}$

The bee's ascent speed can be calculated from Fig. $8 \mathrm{~b}$ in [12], and reaches a value of $\sim 2 \mathrm{~m} / \mathrm{s}$ as well. The bee's descent speed measured during landing maneuvres reaches a value of $2 \mathrm{~m} / \mathrm{s}$ (Fig. $6 \mathrm{~d}$ in [12]). The descent speed isquite similar to our own predictions (8). In order to limit this value $\left(\left|V_{z}\right| \leq 2 \mathrm{~m} / \mathrm{s}\right)$, we limited the stroke amplitude to $|\Delta \Phi| \leq 18^{\circ}$.

\section{SiMULATION SET-UP}

\section{A. Simulated $3 D$ environment}

The simulated $3 \mathrm{D}$ visual environment consists of a straight or tapered flight tunnel (6-meter long, 1-meter wide, and 1meter heigh), the four walls of which are wallpapered with high resolution natural panoramic scene photographs [34] Images were converted into 256 grayscale levels and resized while keeping their original ratio. One image pixel corresponds to one millimeter of the simulated environment (Fig. 2). The four natural grayscale images are shown in Fig. 2: right wall (Fig. 2a), left wall (Fig. 2b), ground (Fig. 2c), and ceiling (Fig. 2d).

\section{B. Optic flow (OF) generated by the bee's own motion}

The bee flies at a speed vector $\vec{V}$ along the flight tunnel covered with natural-scene textures (Fig. 2). The simulated
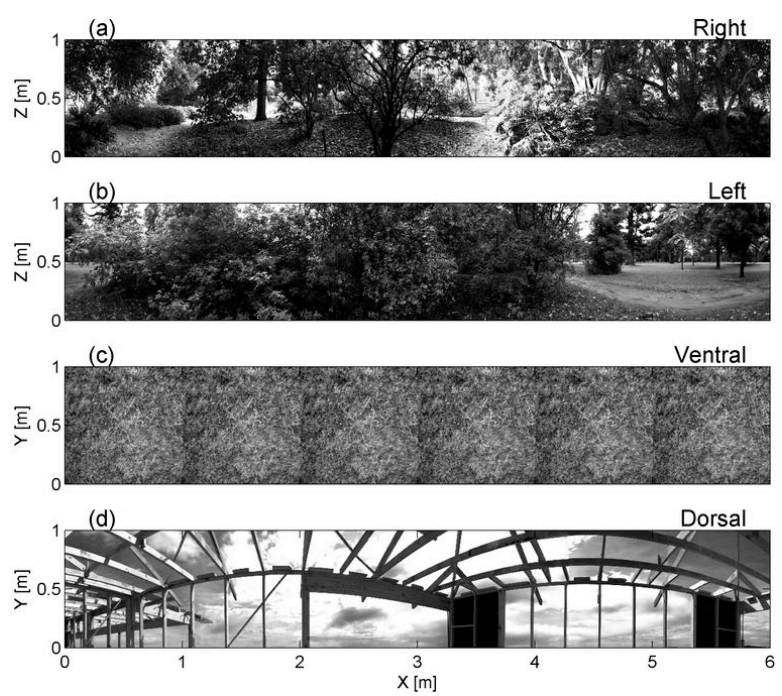

Fig. 2. The grayscale natural scenes used to wallpaper the 4 faces of the simulated tunnel. Resolution of the images is $1000 \times 6000$ pixels $\left(1\right.$ pixel $\left.=1 \mathrm{~mm}^{2}\right)$. Images are therefore $1 \times 6$-meter in size. All four faces of the tunnel are wallpapered with different images: right wall (a), left wall (b), ground (c), and ceiling (d).

bee is assumed to stabilize its gaze by compensating for any body rotations, in the same way as the blowfly does [19]. The bee's head orientation is therefore assumed to be locked to the tunnel X-axis. Since any rotation is compensated for, each OF sensor will receive a purely translational OF, which is the angular velocity of the environmental features projected onto both lateral and vertical (diametrically opposed) OF sensors (Fig. 3).

Each OF sensor receives its own OF, which can be a right OF $\left(\omega_{R}\right)$, a left OF $\left(\omega_{L}\right)$, a ventral OF $\left(\omega_{V}\right)$, or a dorsal OF $\left(\omega_{D}\right)$. The translational OF can be defined simply by the forward speed / distance ratio according to (9).

$\omega_{i}=V_{x} / D_{i}$, with $i \in\{\mathrm{R}, \mathrm{L}, \mathrm{V}, \mathrm{D}\}$

where $V_{x}$ is the bee's forward speed, $D_{R}, D_{L}$, the distances to the lateral (right and left) walls, $D_{V}, D_{D}$, the distances to the ground (ventral eye) and to the ceiling (dorsal eye), respectively (Fig. 3).

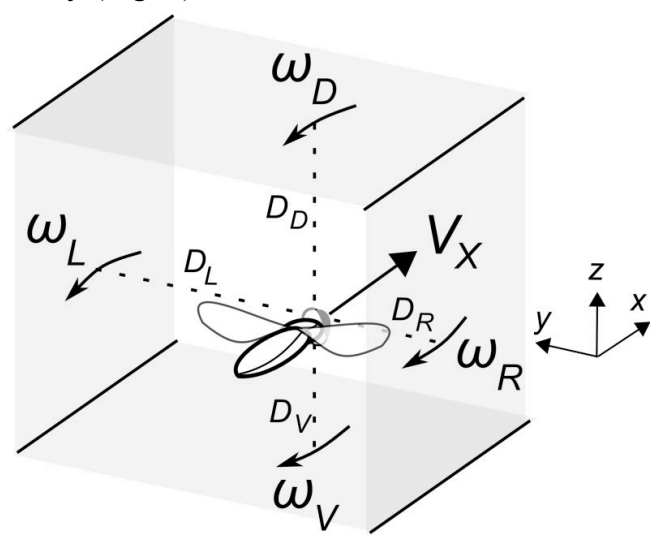

Fig. 3. A simulated bee flying at forward speed $V_{x}$ along a tunnel generates an OF (9) that depends on the perpendicular distance (right $D_{R}$, left $D_{L}$, ventral $D_{V}$, dorsal $D_{D}$ ) from the tunnel surfaces. The simulated bee is equipped with four OF sensors. The sensors'axes are maintained oriented at fixed roll and pitch orientations, perpendicular to the walls, ground and ceiling, respectively, and measure $\mathrm{OF}$ generated laterally $\left(\omega_{L}\right.$ and $\left.\omega_{R}\right)$, ventrally $\left(\omega_{V}\right)$ and dorsally $\left(\omega_{D}\right)$. 


\section{OF sensors onboard the simulated bee}

Two lateral OF sensors and two vertical OF sensors are mounted in diametrically opposed directions, at right angle with respect to the simulated bee symmetry axis. Each OF sensor consists of only two photoreceptors (two pixels) driving an Elementary Motion Detector (EMD). The visual axes of the two photoreceptors are separated by an interreceptor angle $\Delta \varphi=4^{\circ}$. Each photoreceptor angular sensitivity is a bell-shaped function with an acceptance angle (angular width at half height) $\Delta \rho=4^{\circ}$ as well $(\Delta \rho / \Delta \varphi=1)$. Each photoreceptor covers a field of view of $10.4^{\circ} \times 10.4^{\circ}$. The photoreceptor output is computed at each time step (1ms) by convolving the natural scene (Fig. 2) with a 2D Gaussian filter that mimics the photoreceptor Gaussian sensitivity. The principle of the bio-inspired OF sensor developed by Franceschini's research group has been previously described in detail [35-37]. This OF sensor responds as a monotonic function of the angular velocity within a 10 -fold range (from $40 \%$ s to $400 \%$ s) [2], much like the Velocity-Tuned motion-sensitive descending neurons found in honeybees (VT neurons: [38]).

\section{THE ALIS AUTOPILOT}

The simulated bee is controlled by an autopilot called ALIS (ALIS stands for AutopiLot using an Insect-based vision System), which is reminiscent of both OCTAVE autopilot for ground avoidance [2] and LORA III autopilot for speed control and lateral obstacle avoidance [1]. ALIS autopilot relies, however, on four OFs measurement: ventral, dorsal, right, and left. We designed the ALIS autopilot assuming that both speed control and obstacle avoidance issues could a similar solution in the horizontal and vertical planes. The ALIS consists of two visuomotor feedback loops: the speed control loop (along the surge axis) and the positioning control loop (along both the sway and heave axes). Both loops operate in parallel and are intertwined. Each of them involves multiple processing stages (Fig. 4), each one has its own OF set-point: the forward OF set-point and the positioning $O F$ set-point.

In this dual control system, neither speed nor distance from the substrates (walls, ground, or ceiling) need to be measured. The simulated bee will react to any changes in surrounding OFs by adjusting selectively the three orthogonal components $V_{x}, V_{y}$, and $V_{z}$ of its speed vector $\vec{V}$.

The speed control relies on comparing the sums of the OFs in both the horizontal and vertical planes. This loop adjusts the forward speed $V_{x}$ (via the surge dynamics) to keep whichever sum is maximum equal to the forward $O F$ set-point. The positioning control loop relies on whichever of the four OFs measured is greatest. This loop adjusts either the bee's side thrust (2b) or vertical lift (2a) to keep whichever of the four OFs is maximum equal to the positioning OF set-point. The surge (Proportional-Integral, $\mathrm{PI}$ ) and positioning (Proportional-Derivative, PD) controllers were tuned using the same procedures as described for the LORA III autopilot [1].

\section{A. Speed control}

More specifically, the speed control loop aims at holding the maximum of the sums of the two diametrically opposed OF (measured in the horizontal and vertical planes, respectively) constant and equal to a forward OF set-point

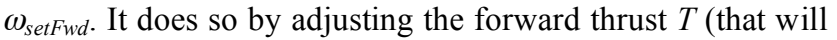
determine the forward speed $V_{x}$ ). In other words, the criterion for regulation here consists in first determining the maximum value beeween the sum of measured OFs in the horizontal plane $\left(\omega_{\text {Rmeas }}+\omega_{\text {Lmeas }}\right)$ and in the vertical plane $\left(\omega_{\text {Vmeas }}+\omega_{\text {Dmeas }}\right)$. The one sum that is the higher is then compared to the forward OF set-point $\omega_{\text {setFwd }}$ (blue loop, Fig. 4). The forward $O F$ set-point is set at $\omega_{\text {setFwd }}=4.57 \mathrm{~V}$ (i.e., $540^{\circ} / \mathrm{s}$ ), a value that is borrowed from that observed in free

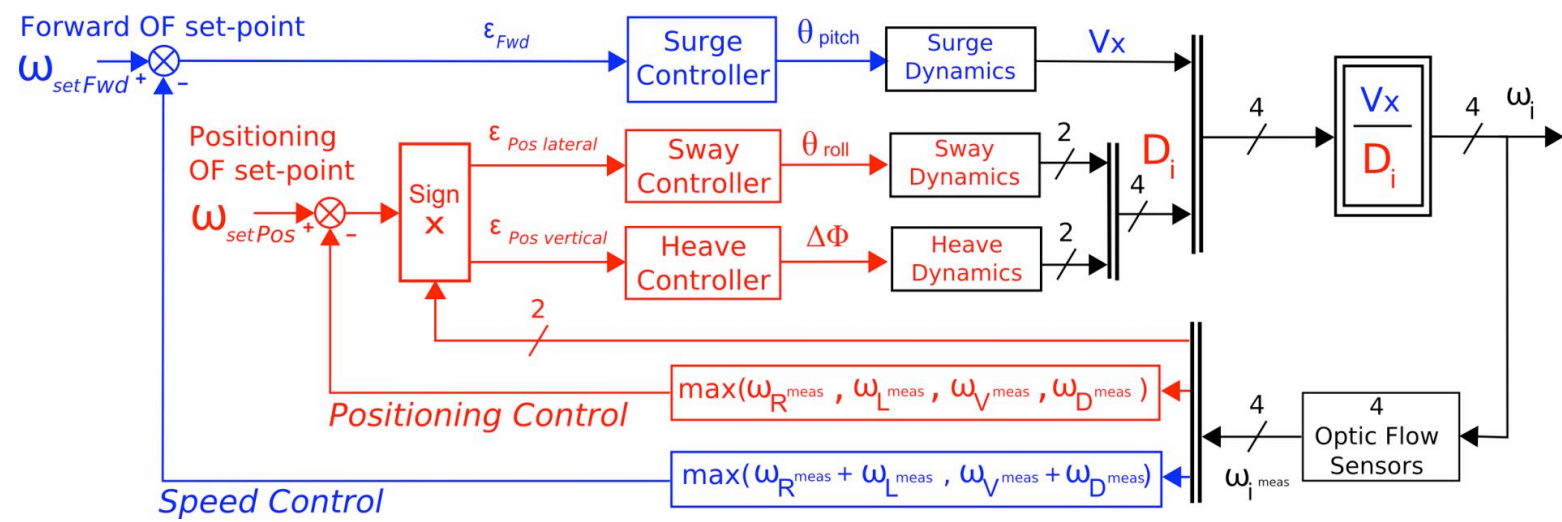

Fig. 4. The ALIS autopilot is based on two intertwined visual feedback loops with their own OF set-point: a speed control loop (in blue) and a positioning control loop (in red). The forward controller (Proportional-Integral PI) adjusts the pitch angle $\theta_{\text {pitch }}$ (that determines $V_{x}$ according to a surge dynamics) on the basis of the maximum of the sums of the two coplanar OF measured. This value is compared to the forward OF set-point $\omega_{\text {setFwd. }}$ The forward controller commands the forward speed so as to minimize the error $\varepsilon_{F w d}$. The sway (or heave) controller adjusts the roll angle $\theta_{\text {roll }}$ (or the stroke amplitude $\Delta \Phi$ ) that will determine the speed $V_{y}$ (or $V_{z}$ ) according to the sway (or heave) dynamics on the basis of the higher OF measured, respectively. The latter value is compared to the positioning OF set-point $\omega_{\text {setPos. }}$. At any time, the direction of avoidance is given by the sign of the difference between the two coplanar OFs measured (horizontal or vertical). The sway (or heave) controller (Proportional-Derivative, PD) commands the sway (or heave) dynamics so as to minimize the error $\varepsilon_{\text {Pos lateral }}$ (or $\varepsilon_{\text {Pos vertical }}$ ), respectively. 
flying bees (Baird et al., 2005). The error signal $\varepsilon_{F w d}$ (the input to the surge controller) is calculated as follows:

$\varepsilon_{F w d}=\omega_{\text {setFwd }}-\max \left[\left(\omega_{\text {Rmeas }}+\omega_{\text {Lmeas }}\right), \quad\left(\omega_{\text {Vmeas }}+\omega_{\text {Dmeas }}\right)\right]$ (10)

The surge dynamics $G_{V x}(s)$ (6a) that relates the bee's forward speed $V_{x}$ to the control signal $\theta_{\text {pitch }}$ is described by a transfer function approximated by a first-order low-pass filter with a time constant of $0.22 \mathrm{~s}(6 \mathrm{a})$.

\section{B. Positioning control}

The positioning control loop is in charge of positioning the bee with respect to either the lateral walls or the ground or the ceiling. Whether positioning is carried out by the sway controller or the heave controller simply depends on whether the maximum OF measured is in the horizontal or vertical plane. The criterion for regulation here is the maximum value of the four OFs measured $\left(\max \left(\omega_{\text {Rmeas }}, \omega_{\text {Lmeas }}, \omega_{\text {Vmeas }}, \omega_{\text {Dmeas }}\right)\right.$, red loop in Fig. 4$)$, i.e., the value given by the nearest substrate (either wall, ground, or ceiling). This $\mathrm{OF}$ regulator is designed to keep the highest OF measured equal to the positioning OF set-point $\omega_{\text {setPos }}$. The criterion is then compared to $\omega_{\text {setPos }}$. The positioning OF set-point is set at $\omega_{\text {setPos }}=2.4 \mathrm{~V}$ (i.e., $315^{\circ} / \mathrm{s}$ ), a value that is again borrowed from that observed in free flying bees (Baird et al., 2005). Notice that the sway or heave dynamics may be alternatively driven, depending on whichever (lateral or vertical) $\mathrm{OF}$ is maximum at any given time. The input to the sway or the heave controller that is not commanded is set to zero $\left(\varepsilon_{\text {Pos lateral }}=0\right.$ or $\left.\varepsilon_{\text {Pos vertical }}=0\right)$.

A Sign function (Fig. 4) automatically selects the substrate to be followed (wall, ground or ceiling). The bee reacts to any deviation in the measured OF by adjusting either the roll angle $\theta_{\text {roll }}$ (sway axis) or the stroke amplitude $\Delta \Phi$ (heave axis), which will determine the bee's lateral speed $V_{y}$ or vertical speed $V_{z}$, respectively (leading to a change in position with respect to the nearest substrate). The error signal $\varepsilon_{P o s}$ (i.e., the input to the sway or heave controller) is computed as follows:

-- If the OF that turns out to be selected is in the horizontal plane $\left(\omega_{\text {Lmeas }}\right.$ or $\left.\omega_{\text {Rmeas }}\right)$, the input of the sway controller will be:

$\varepsilon_{\text {Pos lateral }}=\operatorname{sign}\left(\omega_{\text {Lmeas }}-\omega_{\text {Rmeas }}\right) \times\left(\omega_{\text {setPos }}-\max \left(\omega_{\text {Rmeas }}, \omega_{\text {Lmeas }}\right)\right.$

and the input to the heave controller will be $\varepsilon_{\text {Pos vertical }}=0$.

-- If the OF that turns out to be selected is in the vertical plane $\left(\omega_{\text {Dmeas }}\right.$ or $\left.\omega_{\text {Vmeas }}\right)$, the input to the heave controller will be:

$\varepsilon_{\text {Pos vertical }}=\operatorname{sign}\left(\omega_{\text {Dmeas }}-\omega_{\text {Vmeas }}\right) \times\left(\omega_{\text {setPos }}-\max \left(\omega_{\text {Dmeas }}, \omega_{\text {Vmeas }}\right)\right.$

and the input to the sway controller will be $\varepsilon_{\text {Pos lateral }}=0$.

\section{V.SIMULATION RESULTS}

\section{A. Automatic tunnel-following}

In figure 5, the simulated environment is a straight tunnel (6-meter long, 1-meter wide, and 1-meter heigh). Fig. 5a shows a perspective view. Walls, ground, and ceiling are wallpapered using natural grayscale images (Fig. 2). The simulated bee enters the tunnel at speed $V_{x o}=0.2 \mathrm{~m} / \mathrm{s}$ and initial coordinates $x_{0}=0.1 \mathrm{~m}, y_{0}=0.15 \mathrm{~m}, z_{0}=0.15 \mathrm{~m}$ (Fig. $5 \mathrm{~b}$ ). Fig. $5 \mathrm{c}$ shows the trajectory in the horizontal plane $(x, y)$ gand Fig. $5 \mathrm{~d}$ in the vertical plane $(x, z)$.

The simulated bee can be seen to gradually increase both its height of flight (Fig. 5d) and its right clearance (Fig. 5c) to $0.33 \mathrm{~m}$, while the forward speed (Fig. 5e) automatically increases to $2 \mathrm{~m} / \mathrm{s}$ (i.e., the maximal speed allowed).

By selecting the highest value of the four EMD ouptuts
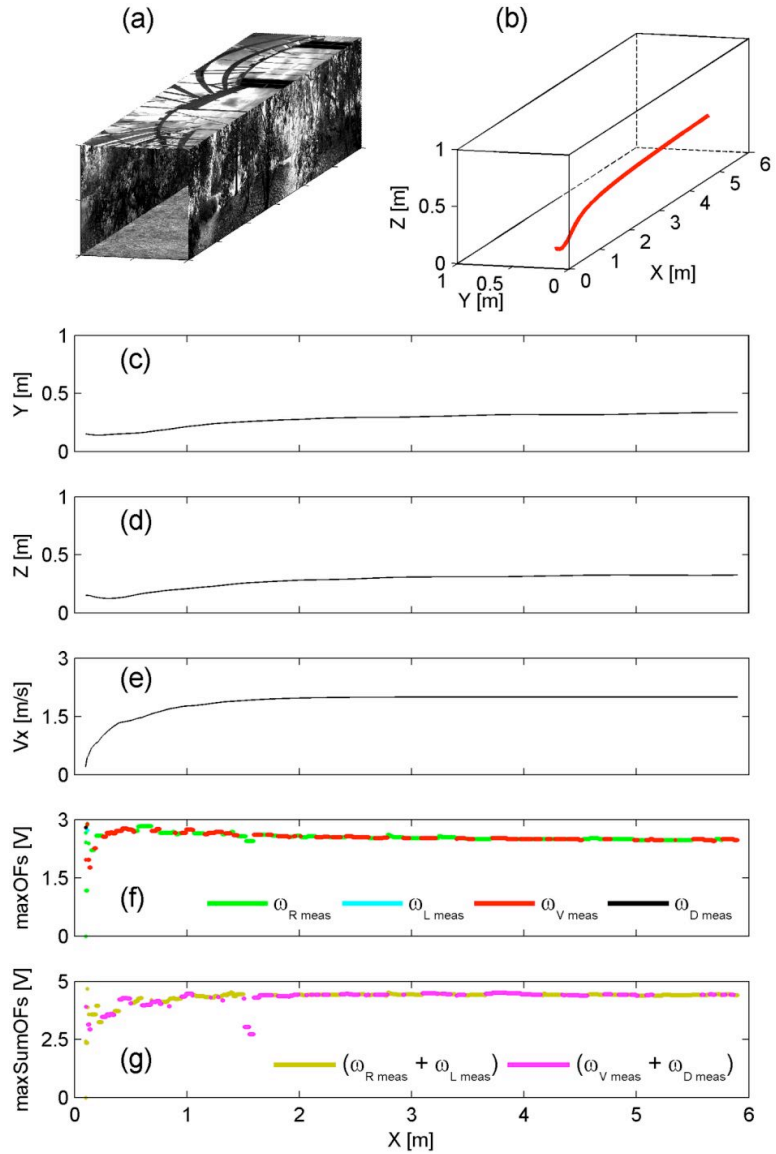

Fig. 5. (a) Perspective view of the straight flight tunnel. (b) Simulated bee's 3D trajectory starting at $x_{0}=0.1 \mathrm{~m}, y_{0}=0.15 \mathrm{~m}, z_{0}=0.15 \mathrm{~m}$ with initial speed $V_{x o}=0.2 \mathrm{~m} / \mathrm{s}$. (c) Flight track in the horizontal plane $(x, y)$. (d) Trajectory in the vertical plane $(x, z)$. (e) Forward speed $V_{x}$ profile. (f) Positioning feedback signal equal to the maximum output of the four OFs sensors: right $\mathrm{OF}$ sensor = green; left $\mathrm{OF}$ sensor = cyan; ventral OF sensor $=$ red; dorsal OF sensor $=$ black. $(\mathrm{g})$ Forward feedback signal equal to the maximum of the sum of the two coplanar OFs measured (horizontal OF sensors = yellow; vertical OF sensors = magenta).

(Fig. 5f), the positioning control loop happends to command either the heave or sway dynamics at a time making the bee avoid both the ground and the right wall. In the steady state, the simulated bee can be seen to reach a ventral and a right OF measured $\left(\omega_{\text {Vmeas }}=\omega_{\text {Rmeas }}=2.48 \mathrm{~V}\right.$, i.e., $\left.355^{\circ} / \mathrm{s}\right)$ that are both close to the positioning OF set-point $\left(\omega_{\text {setPos }}=2.4 \mathrm{~V}\right.$, i.e., $315 \%$ s). The speed achieved is close to saturation. The forward feedback signal reaches $4.42 \mathrm{~V}\left(525^{\circ} / \mathrm{s}\right)$ in Fig. $5 \mathrm{~g}$, 
which is close to the forward OF set-point $\left(\omega_{\text {setFwd }}=4.57 \mathrm{~V}\right.$, i.e., $540 \%$ s).

Taken together, these results show that the ALIS autopilot makes the simulated bee:

-- adopt a certain cruise speed

-- keep a certain clearance from the substrates (wall+ground) thus automatically generating both terrainfollowing and wall-following behaviors.

\section{B. Effect of the local absence of $O F$}

Figure 6 tests the simulated bee in the local absence of contrast on the left wall or ceiling of the tunnel (Fig. 6a). These "no contrast" zones could be either a true aperture or a lack of texture (Fig. 6a). The simulated bee is made to enter the tunnel at speed $V_{x o}=0.2 \mathrm{~m} / \mathrm{s}$ and at initial coordinates $x_{0}=0.1 \mathrm{~m}, y_{0}=0.85 \mathrm{~m}, z_{0}=0.85 \mathrm{~m}$ (Fig. $6 \mathrm{~b}$ ). Fig. $6 \mathrm{c}$ shows the trajectory in the horizontal plane $(x, y)$ and Fig. $6 \mathrm{~d}$ in the vertical plane $(x, z)$.

It can be seen from Fig. 6 that the simulated bee is not dramatically disturbed neither by the 2-meter long aperture seen on its left-hand side (at the beginning of the tunnel), nor by a similar aperture in its dorsal field of view (at the end of the tunnel).

The forward feedback signal (Fig. 6g) can be seen to select either vertical or horizontal EMD outputs in case of the lateral or vertical absence of EMD outputs corresponding here to the "no contrast" zones (from $X=0.5 \mathrm{~m}$ to $X=2.5 \mathrm{~m}$ and from $X=3.5 \mathrm{~m}$ to $X=5.5 \mathrm{~m}$ ). This forward feedback signal selection allows the simulated bee to maintain a relatively constant speed $V_{x}=1.85 \mathrm{~m} / \mathrm{s}$ throughout its journey (Fig. 6e).

The positioning feedback signal (Fig. 6f) can be seen to select either the left or the dorsal EMD outputs in case of the lateral or vertical absence of EMD outputs corresponding here to the "no contrast" zones (from $X=0.5 \mathrm{~m}$ to $X=2.5 \mathrm{~m}$ and from $X=3.5 \mathrm{~m}$ to $X=5.5 \mathrm{~m}$ ). The positioning feedback signal automatic selection allows the simulated bee to maintain a dorsal clearance $D_{D}=0.35 \mathrm{~m}$ (Fig. 6d) and a left clearance $D_{L}=0.39 \mathrm{~m}$ (Fig. $6 \mathrm{c}$ ) throughout its journey.

These results show that the ALIS autopilot makes the simulated bee cross the tunnel, without being dramatically disturbed by the lateral or ventral "no contrast" zone.

C. Automatic deceleration and acceleration in a tapered tunnel.

The simulated tunnel here is a 6-meter long, 1-meter heigh tapered tunnel with a 1-meter wide entrance and a 0.25 meter wide constriction located midway (Fig. 7a). This tunnel is designed to test the ALIS autopilot in its ability to reject a strong lateral OF disturbance. The simulated bee enters the tunnel at speed $V_{x o}=0.2 \mathrm{~m} / \mathrm{s}$ and at initial coordinates $x_{0}=0.1 \mathrm{~m}, y_{0}=0.85 \mathrm{~m}, z_{0}=0.6 \mathrm{~m}$ (Fig. $7 \mathrm{~b}$ ). Fig. $7 \mathrm{c}$ shows the trajectory in the horizontal plane $(x, y)$ and Fig. $7 d$ in the vertical plane $(x, z)$.

The simulated bee can be seen to automatically slow down as it approaches the narrowest section of the tapered tunnel, and to accelerate again when the tunnel widens beyond it (Fig. 7e). The positioning feedback signal (Fig. 7f) can be seen to have selected the left measured OF, which appears to be maintained close to the positioning OF set-point
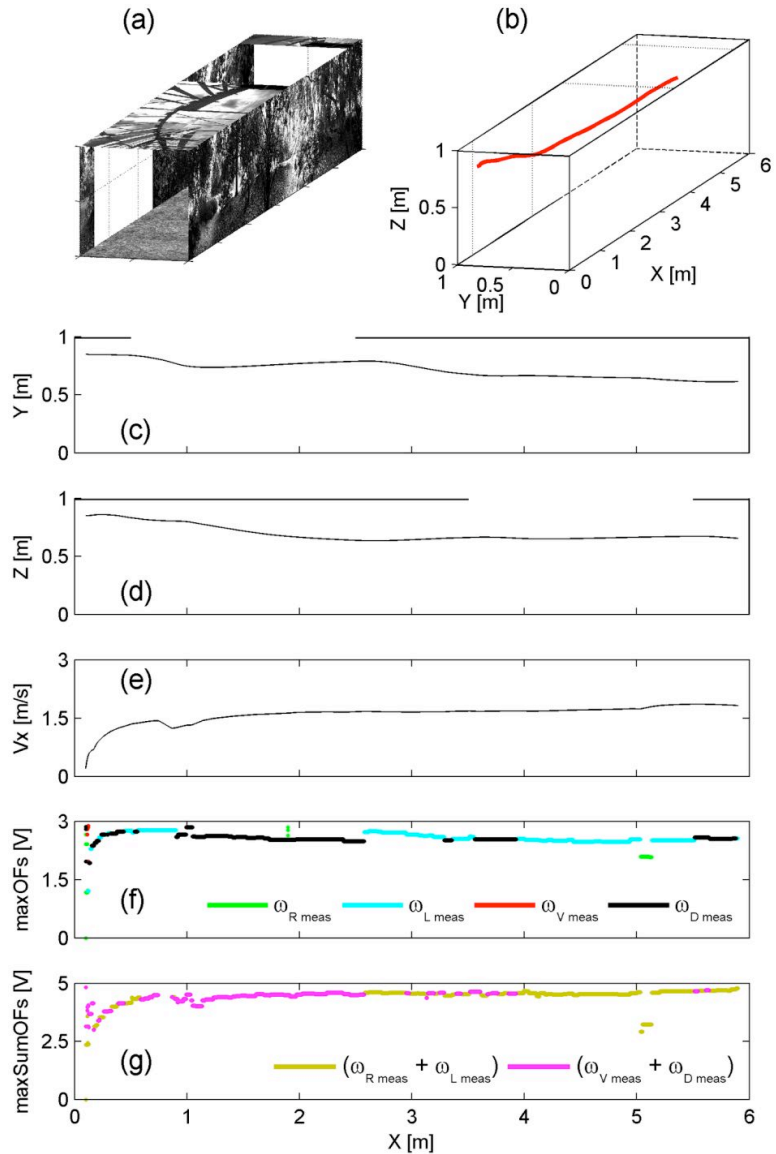

Fig. 6. (a)Perspective view of the straight flight tunnel covered by two "no contrast" zones. (b)The simulated bee's 3D trajectory starting from $x_{0}=0.1 \mathrm{~m}, y_{0}=0.85 \mathrm{~m}, z_{0}=0.85 \mathrm{~m}$, at the forward speed $V_{x o}=0.2 \mathrm{~m} / \mathrm{s}$. (c)Trajectory in the horizontal plane $(x, y)$. (d)Same trajectory in the vertical plane $(x, z)$. (e)Forward speed $V_{x}$ profile. (f)The positioning feedback signal determined by the maximum output of the four OFs sensors (right OF sensor = green color; left OF sensor $=$ cyan color; ventral OF sensor $=$ red color; dorsal OF sensor $=$ black color). (g)The forward feedback signal determined by the maximum of the sum of the two coplanar OFs measured (horizontal OF sensors $=$ yellow color; vertical OF sensors $=$ magenta color $)$.

throughout the trajectory (Fig. 7f). The simulated bee is seen to follow the left wall of the tapered tunnel. The reason is simply due to the fact that its initial position was close to that wall. As the tunnel narrows only in the horizontal plane, the OF in the vertical plane is of little concern to the ALIS autopilot (Fig. 7g).

The ALIS autopilot makes the simulated bee cross the tapered tunnel (tapering angle $7^{\circ}$ ), without being dramatically disturbed by a major bilateral OF disturbance.

\section{CONCLUSION}

We have presented an OF-based 3D autopilot, called ALIS. Results show that a bee equipped with the ALIS autopilot can navigate safely under visual control along a straight tunnel (Fig. 5). This holds even when the wall or the ceiling is locally devoid of texture (Fig. 6) and when the tunnel narrows or expands (Fig. 7). These feats are all achieved with a really minimalistic visual system, which 
(a)

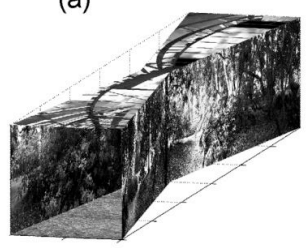

(b)

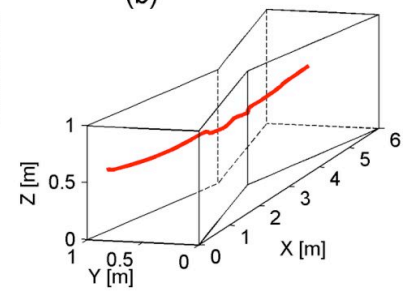

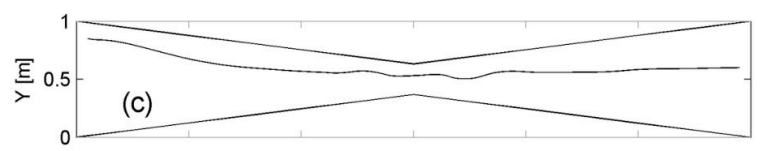
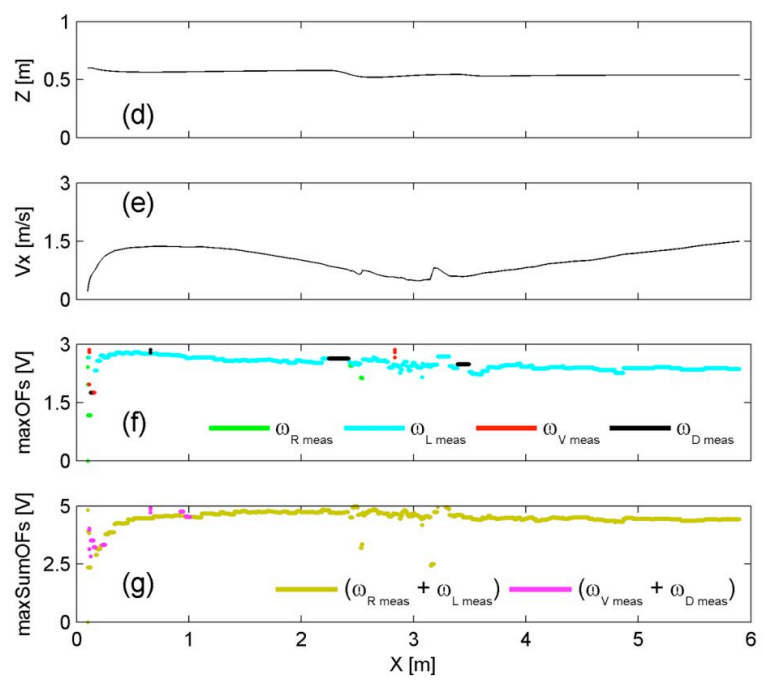

Fig. 7. (a) Perspective view of the tapered tunnel. (b) The simulated bee's $3 \mathrm{D}$ trajectory starting at initial coordinates $x_{0}=0.1 \mathrm{~m}, y_{0}=0.85 \mathrm{~m}$, $z_{0}=0.6 \mathrm{~m}$, and at speed $V_{x o}=0.2 \mathrm{~m} / \mathrm{s}$. (c) Trajectory in the horizontal plane $(x, y)$. (d) Trajectory in the vertical plane $(x, z)$. (e) Forward speed $V_{x}$ profile. (f) The positioning feedback signal determined by the maximum output of the four OFs sensors (right $\mathrm{OF}$ sensor $=$ green; left $\mathrm{OF}$ sensor = cyan; ventral OF sensor = red; dorsal OF sensor = black). (g) The forward feedback signal determined by the maximum of the sum of the two coplanar OFs measured (horizontal OF sensors $=$ yellow; vertical OF sensors = magenta).

consists of only eight pixels forming four EMDs (one pair oriented horizontally, one pair vertically). Key to the working of the ALIS autopilot is a pair of OF regulators that aim at maintaining the perceived OF constant by acting upon the forward, lateral, and vertical thrusts. Specifically, these two OF regulators operates as follows:

1) The first $O F$ regulator adjusts the bee's forward speed so as to keep the maximum of the sums of the two opposite OFs (i.e., left+right and ventral+dorsal) equal to a forward OF set-point. The outcome is that the bee's forward speed becomes proportional to the narrower dimension (width or height) of the corridor (Fig. 7e). Further simulations showed (data not shown) that this holds regardless of the bee's initial position at the tunnel entrance. The forward speed attained by the simulated bee depends upon the forward OF set-point $\omega_{\text {setFwd }}$.

2) The second $O F$ regulator adjusts the bee's lateral and vertical position so as to keep the highest $O F$ value (from whatever substrate: walls, ground, or ceiling) equal to

the positioning OF set-point. The outcome is that the clearance from the nearest wall (or ground or ceiling) becomes proportional to the bee's forward speed as defined in 1). The clearance from the nearest wall (or ground or ceiling) depends upon the positioning OF set-point $\omega_{\text {setPos }}$.

The great advantage of this visuomotor control system is that it operates without any needs for explicit knowledge of speed and distance, and hence without any needs for velocimeters and range sensors. Behavior is of primary concern, not metrics: the simulated bee behaves sensibly although it is completely 'unaware' of its ground speed and distances from the walls, ground, and ceiling. The simulated bee navigates on the basis of two parameters only: the forward OF set-point $\omega_{\text {setFwd }}$ and the positioning OF set-point $\omega_{\text {setPos }}$ (Fig. 4). The ALIS explicit control scheme proposed here (Fig. 4) is in line with the ecological approach [3], in which an animal's vision system is thought to drive the locomotory system directly, without using any "representation" of the environment (see also [14]). The ALIS control scheme (Fig. 4) accounts remarkably well for behaviors observed in real bees flying along a stationary corridor [5-6,10] or along a tapered corridor [8], despite the very low number (four) of OF sensors with which it is equipped (one on the right, one on the left, one underneath, and one on the top: Fig. 3). One may reasonably assume that bees are equipped with an ALIS-like dual OF regulator - a control system that is, in addition, little demanding in terms of neural conputation.

In terms of applications, an ALIS autopilot would provide the vehicle on which it would be mounted with both a cruise control system and an anti-collision system. ALIS could be applied to vehicles in which the three translational dynamics are uncoupled such as MAVs (e.g.: conventional, coaxial, or quadrotor mini-helicopters) and underwater vehicles (e.g., AUVs and ROVs).

Insect-based visuomotor control systems can yield solutions requiring a much smaller number of pixels than those used in present-day computer-vision systems harnessed to mobile robots. The ALIS autopilot presented here may open the way to lightweight and low-cost visual guidance systems for autonomous vehicle navigation in unfamiliar indoor environments, as well as in natural or urban canyons where GPS signals may be considerably attenuated by the presence of rocks or buildings. The nonemissive OF sensors and the simple processing system described here are particularly suitable for use on MAVs, whose small size imposes draconian constraints on avionic payload and onboard energy resources.

\section{ACKNOWLEDGMENTS}

We thank S. Viollet and L. Kerhuel for their fruitful comments and suggestions during this work, R. Brinkworth and D. O'Carroll (Adelaide Uni., Australia) for kindly making their panoramic images avaible to us. 


\section{REFERENCES}

[1] J. Serres, D. Dray, F. Ruffier, and N. Franceschini, "A vision-based autopilot for a miniature air-vehicle: joint speed control and lateral obstacle avoidance," Autonomous Robot, vol. 25 pp. $103-122,2008$.

[2] F. Ruffier and N. Franceschini, "Optic flow regulation: the key to aircraft automatic guidance," Robotics and Autonomous Systems, vol. 50(4), pp. 177-194, 2005.

[3] J.J. Gibson, "The perception of the visual world," Boston: Houghton Mifflin, 1950.

[4] G.A. Horridge, "The evolution of visual processing and the construction of seeing system," Proc. Roy. Soc. Lond. B, vol. 230, pp. 279-292, 1987

[5] M.V. Srinivasan, M. Lehrer, W.H. Kirchner, and S.W. Zhang, "Range perception through apparent image speed in freely flying honeybees," Vis. Neurosci., vol. 6, pp. 519-535, 1991

[6] J. Serres, F. Ruffier, G.P. Masson, and N. Franceschini, "A bee in the corridor: centering or wall-following?, "In Proc. of the $7^{\text {th }}$ meeting of the German neuroscience society - $31^{\text {st }}$ Göttingen neurobiology conference, Göttingen, Germany, T14-8B, 2007.

[7] R. Preiss, "Motion parallax and figural properties of depth control flight speed in an insect," Biol. Cyb., vol. 57, pp. 1-9, 1987.

[8] M.V. Srinivasan, S.W. Zhang, M. Lehrer, and T.S Collett, "Honeybee navigation. en route to the goal: visual flight control and odometry," J. Exp. Biol., vol. 199, pp. 237-244, 1996.

[9] E. Baird, M.V. Srinivasan, S. Zhang, and A. Cowling, "Visual control of flight speed in honeybees, “ J. Exp. Biol., vol. 208, pp. 3895-3905 2005.

[10] E. Baird, M.V. Srinivasan, S. Zhang, R. Lamont, and A. Cowling, "Visual control of flight speed and height in honeybee," LNAI, vol. 4095, pp. 40-51, 2006.

[11] N. Franceschini, F. Ruffier, and J. Serres, "A bio-inspired flying robot sheds light on insect piloting abilities," Current Biology, vol. 17, pp. 329-335, 2007.

[12] M. V. Srinivasan, S.W. Zhang, J.S. Chahl, E. Barth, and S.Venkatesh, "How honeybees make grazing landings on flat surfaces," Biol. Cyb. vol. 83, pp. 171-183, 2000.

[13] D. Coombs and K. Roberts, "Bee-bot: using peripheral optical flow to avoid obstacle," in SPIE: Intelligent robots and computer vision XI, vol. 1825, pp. 714-721, 1992.

[14] A.P. Duchon and W.H. Warren, "Robot navigation from a Gibsonian viewpoint," in Proc. Int. Conf. Syst. Man and Cyb., pp. 2272-2277, San Antonio, Texas, 1994.

[15] J. Santos-Victor, G. Sandini, F. Curotto, and S. Garibaldi, "Divergent stereo in autonomous navigation: from bees to robots," Int. J. of Comp. Vision, vol. 14, pp. 159-177, 1995.

[16] K. Weber, S. Venkatesh, and M.V. Srinivasan, "Insect inspired behaviours for the autonomous control of mobile robots, " in M.V. Srinivasan and S. Venkatesh (Eds), From living eyes to seeing machines, Oxford: Oxford University Press, 1997.

[17] J.S. Humbert, A. Hyslop, and M. Chinn, "Experimental validation of wide-field integration methods for autonomous navigation, "In Proc. IEEE int. conf. intelligent robots and systems, IROS, 2007.

[18] C.P. Ellington, "The aerodynamics of hovering insect flight. III. Kinematics," Phil. Trans. Roy. Soc. Lond. B, vol. 305, pp. 41-78, 1984.

[19] C. Schilstra and J.H. van Hateren, "Blowfly flight and optic flow. I. Thorax kinematics and flight dynamics," J. Exp. Biol., vol. 202, pp. 1481-1490, 1999.

[20] T.R. Neumann and H.H. Bülthoff, "Insect visual control of translatory flight," In Proceedings of the $6^{\text {th }}$ european Conf. on Artificial Life ECAL, LNCS/LNAI, vol. 2159, pp. 627-636, 2001.

[21] J.-C. Zufferey and D. Floreano, " Toward 30-gram autonomous indoor aircraft: vision-based obstacle avoidance and altitude control, " in Proc. IEEE int. conf. on robotics and automation (ICRA), pp. 25942599, Barcelona, Spain, 2005.

[22] A. Beyeler, J.-C. Zufferey, and D. Floreano, "3D vision-based navigation for indoor microflyers," in Proc. IEEE int. conf. on robotics and automation, ICRA, pp. 1336-1341, 2007.

[23] W.B. Dickson, A.D. Straw, C. Poelma, and M.H. Dickinson, "An integrative model of insect flight control," In Proceeding of the $44^{\text {th }}$ AIAA aerospace sciences meeting and exhibit, AIAA-2006-0034, 2006.
[24] K. von Frisch, "Gelöste und ungelöste rätsel der bienen-sprache," Naturwissenschaften, vol. 35, pp. 38-43, 1948.

[25] J.R. Riley, et al. , "The Automatic Pilot of Honeybees," Proc. Roy. Soc. Lond., vol. 270,pp. 2421-2414, 2003.

[26] C. David, "The relationship between body angle and flight speed in free-flying Drosophila," Physiol. Entomol., vol. 3, pp. 191-195, 1978.

[27] W. Nachtigall, R. Widmann, and M. Renner, "Uber den ortsfesten freien Flug von Bienen in einem Saugkanal," Apidologie, vol. 2, pp. 271-282, 1971 .

[28] H. Esch, W. Nachtigall, and S.N. Kogge, "Correlations between aerodynamic output, electrical activity in the indirect flight muscles and wing positions of bees flying in a servomechanically controlled flight tunnel," J. Comp. Physiol., vol. 100, pp. 147-159, 1975.

[29] M.E. Dillon and R. Dudley, "Allometry of maximum vertical force production during hovering flight of neotropical orchid bees (Apidae: Euglossini)," J. Exp. Biol., vol. 207, pp. 417-425, 2004.

[30] D.L. Altshuler, W.B. Dickson, J.T. Vance, S.P. Roberts, and M.H. Dickinson, "Short-amplitude high-frequency wing strokes determine the aerodynamics of honeybee flight," $P N A S$, vol. 102, $\mathrm{n}^{\circ} 50$, pp.18213-18218, 2005.

[31] R. Dudley, "Extraordinary flight performance of orchid bees (apidae: euglossini) hovering in heliox $\left(80 \% \mathrm{He} / 20 \% \mathrm{O}_{2}\right)$," J. Exp. Biol., vol. 198, pp. 1065-1070, 1995.

[32] S.P. Sane and M.H. Dickinson, "The aerodynamic effects of wing rotation and a revised quasi-steady model of flapping flight," J. Exp. Biol., vol. 205, pp.1087-1096, 2002.

[33] R. Dudley, "The biomechanics of insect flight: form, function, evolution," Princeton: Princeton University Press, 2000.

[34] R.S.A. Brinkworth and D.C. O'Carroll, "Biomimetic Motion Detection, " in Proceedings of The International Conference on Intelligent Sensors, Sensor Networks and Information Processing (ISSNIP), vol. 3, pp.137-142, 2007.

[35] C. Blanes, "Appareil visuel élémentaire pour la navigation à vue d'un robot mobile autonome," MS in Neuroscience, Marseille, France: University of Aix-Marseille II, 1986.

[36] M. Pudas, et al., "A miniature bio-inspired optic flow sensor based on low temperature co-fired ceramics(LTCC) technology," Sensors and Actuators A, vol. 133, pp. 88-95, 2007.

[37] F. Aubépart and N. Franceschini, "A bio-inspired optic flow sensor based on FPGA: application to micro-air-vehicles," $J$. of Microprocessors and Microsystems, vol. 31(6), pp. 408-419, 2007.

[38] M.R. Ibbotson, "Evidence for velocity-tuned motion-sentive neurons in the honeybee," Proc. Roy. Soc. Lond. B., vol. 268, pp. 2195-2201, 2001. 